\title{
Contrast Matching of Detergent Micelles for Membrane Protein Studies with SANS
}

\section{Ryan C. Oliver, Sai Venkatesh Pingali and Volker Urban}

Oak Ridge National Lab

Detergents represent the most frequently employed tool to solubilize membrane proteins for structural and functional investigations in solution. However, an excess of empty (protein-free) detergent micelles are often present in addition to the desired protein-detergent complexes. For SANS experiments, which record the ensemble average of particles in solution, the signal from these empty micelles has a negative impact on the scattering of interest. Additionally, the core-shell architecture of micelles produces a structure factor which remains present at the total detergent match point. We present one approach for the rational design of mixed micelles containing a deuterated detergent analog, which produce a negligible contrast between core and shell, and can be fully contrast matched to better resolve membrane protein structure with SANS. 